\title{
A NOTE ON CIRCLE PATTERNS ON SURFACES
}

\author{
REN GUO
}

\begin{abstract}
In this paper we give two different proofs of Bobenko and Springborn's theorem of circle pattern: there exists a hyperbolic (or Euclidean) circle pattern with proscribed intersection angles and cone angles on a cellular decomposed surface up to isometry (or similarity).
\end{abstract}

\section{INTRODUCTION}

In the work of Bobenko and Springborn [1, they produced a variational principle for circle patterns on surfaces in Euclidean and hyperbolic geometry. The goal of this paper is to give two different proofs of Bobenko and Springborn's theorem. First we will give a direct proof of Bobenko and Springborn's theorem in Euclidean and hyperbolic geometry by using the continuity method used by Thurston [16], Marden and Rodin [11. Then we will use another variational principle following Rivin's strategy [12] [13] to prove Bobenko and Springborn's theorem in hyperbolic geometry.

Bobenko and Springborn's proof consists of two steps. In the first step they use the feasible flow to find the necessary and sufficient condition for the existence of an angle structure (i.e.coherent angle system). In the second step they introduced an energy function whose variables are radii of circles and they can show that the unique critical point of the energy function corresponds to circle pattern with prescribed intersection angles and cone angles.

This variational principle is first used by Colin de Verdiére 3 in the proof of Andreev-Thurston's circle packing theorem in Euclidean and hyperbolic geometry. Brägger 2 2 found a new energy function for Euclidean circle packing whose variables are certain angles. This energy function turns out to be the Legendre transformation of Colin de Verdiére's.

To character the cone metric on a triangulated surface, Rivin produced a variational principle. Rivin [13] used the duality theorem in linear programming to find the necessary and sufficient condition for the existence of an angle structure. Rivin [12] introduced an energy function whose variables are certain angles and he can show the unique maximal point of the energy function corresponds to the angle structure induced by a cone metric. Following Rivin's method, Leibon [6] characterized the hyperbolic cone metric. For the spherical cone metric, the angle structure part was solved by the author [4] and the energy function part was solved by Luo [7. We will show that Rivin's strategy also works in the circle pattern problem.

2000 Mathematics Subject Classification. 52C26.

Key words and phrases. circle pattern, continuity method, variational principle.

This work is partially supported by NSF Grant \#0625935. 
Our energy function is the Legendre transformation of the energy function in Bobenko and Springborn [1]. Springborn [14] already found this Legendre transformation. Here we will show this function can be naturally derived from Luo's principle of "the derivative cosine law". Luo 7] established this principle and was able to recover the energy function of Colin de Verdiére, Brägger, Rivin, Leibon. See the related work of Luo [8] [9] [10].

Springborn [15 used Rivin's strategy to solve another circle pattern problem.

To state Bobenko and Springborn's theorem, let's recall some definitions first. Suppose $\Sigma$ is a closed surface with a cell decomposition. Let $E, F$ be the sets of edges and faces of the cell decomposition respectively. If $e \in E, f \in F$, we use $e<f$ to denote that $e$ is an edge of $f$. Suppose we are given a radius function $r: F \rightarrow(0, \infty)$ and an intersection angle function $D: E \rightarrow(0, \pi)$. We will use these data to construct a hyperbolic (or Euclidean) structure on $\Sigma$ with cone points.

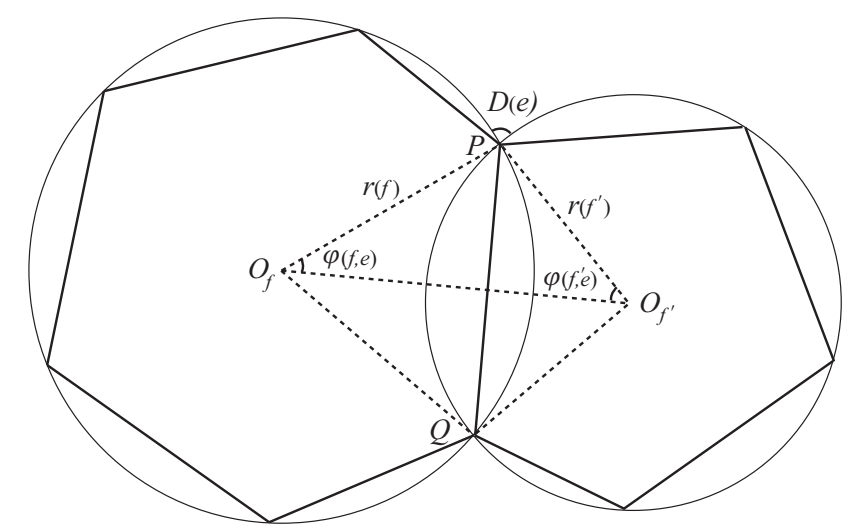

FiguRE 1. Cell decomposition, quadrilateral, circle pattern

First, choose a point $O_{f}$ in the interior of each $f$. As in Figure 1, if the face $f$ and $f^{\prime}$ share an edge $e$ with end points $P$ and $Q$, we realize the quadrilateral $O_{f} P O_{f^{\prime}} Q$ as a hyperbolic (or Euclidean) quadrilateral with edge lengths $\left|O_{f} P\right|=\left|O_{f} Q\right|=$ $r(f),\left|O_{f^{\prime}} P\right|=\left|O_{f^{\prime}} Q\right|=r\left(f^{\prime}\right)$ and angles $\angle O_{f} P O_{f^{\prime}}=\angle O_{f} Q O_{f^{\prime}}=\pi-D(e)$. For each edge $e$ we construct such a quadrilateral. By gluing together all the quadrilaterals, we obtain a hyperbolic (or Euclidean) structure on $\Sigma$ with cone points. Furthermore for each face $f$ there is a circumcircle with center $O_{f}$ and radius $r(f)$. And the intersection angle of the circumcircles of face $f$ and $f^{\prime}$ is $D(e)$. We call this a hyperbolic (or Euclidean) circle pattern. In the hyperbolic (or Euclidean) triangle $O_{f} P O_{f^{\prime}}$, denote the angle at $O_{f}$ by $\varphi(f, e)$. Then the cone angle at point $O_{f}$ is

$$
\sum_{e<f} 2 \varphi(f, e),
$$

where the sum runs over all edges of the face $f$. Thus we obtain a cone angle function $\Phi: F \rightarrow(0, \infty)$.

On the other hand, if we are given an intersection angle function $D: E \rightarrow(0, \pi)$ and a cone angle function $\Phi: F \rightarrow(0, \infty)$, we try to solve the radius function 
$r: F \rightarrow(0, \infty)$, equivalently to obtain a circle pattern. Bobenko and Springborn [1] obtained the following result by using a variational principle. Their result can cover the case of surfaces with boundary. But for simplicity we only consider the closed surfaces in this paper.

For any subset $X \subseteq F$, let $E(X)=\{e \in E \mid e<f \in X\}$ be the set of edges contained in at least one of the faces in $X$.

Theorem 1.1 (Bobenko-Springborn [1] Theorem 3). Given a cellular decomposed surface $\Sigma$, a function $D: E \rightarrow(0, \pi)$ and a function $\Phi: F \rightarrow(0, \infty)$, there exists a unique Euclidean circle pattern with the the intersection angle function $D$ and the cone angle function $\Phi$ if and only if

$$
\sum_{f \in F} \frac{1}{2} \Phi(f)=\sum_{e \in E} D(e)
$$

and

$$
\sum_{f \in X} \frac{1}{2} \Phi(f)<\sum_{e \in E(X)} D(e)
$$

for any nonempty subset $X \subset F, X \neq F$.

Theorem 1.2 (Bobenko-Springborn [1] Theorem 3). Given a cellular decomposed surface $\Sigma$, a function $D: E \rightarrow(0, \pi)$ and a function $\Phi: F \rightarrow(0, \infty)$, there exists a unique hyperbolic circle pattern with the the intersection angle function $D$ and the cone angle function $\Phi$ if and only if

$$
\sum_{f \in X} \frac{1}{2} \Phi(f)<\sum_{e \in E(X)} D(e)
$$

for any nonempty subset $X \subseteq F$.

In section 2 we will prove Theorem 1.1 by using the continuity method. In section 3 we will prove Theorem 1.2 by using the continuity method. In section 4 we will prove Theorem 1.2 by using a variational principle.

\section{Continuity Method For Euclidean Circle Pattern}

Proof of Theorem 1.1. Assume the set of faces of the cell decomposition is $F=$ $\left\{f_{1}, \ldots, f_{n}\right\}$. Due to Euclidean similarity, it is enough to consider the space of radii $\Delta=\left\{\left(r\left(f_{1}\right), \ldots, r\left(f_{n}\right)\right) \mid r\left(f_{1}\right)+\ldots+r\left(f_{n}\right)=1\right\}$ which is a simplex of dimension $n-1$. For a fixed intersection angle function $D: E \rightarrow(0, \pi)$, if the radius function $r: F \rightarrow(0, \infty)$ is given, we can find the cone angle function $\Phi: F \rightarrow(0, \infty)$. Therefore there is a mapping $P: \Delta \rightarrow \mathbb{R}^{n}$ sending the radii $\left(r\left(f_{1}\right), \ldots, r\left(f_{n}\right)\right)$ to its cone angles $\Phi=\left(\Phi\left(f_{1}\right), \ldots, \Phi\left(f_{n}\right)\right)$.

Let $\delta=\left\{\Phi \in \mathbb{R}_{>0}^{n} \mid \Phi\right.$ satisfies the conditions (1.1), (1.2) $\}$ be an $n-1$ dimensional polytope in $\mathbb{R}^{n}$. We claim that $P(\Delta) \subset \delta$. We need to check that, if $\Phi \in P(\Delta), \Phi$ satisfies the conditions (1.1), (1.2). 
In fact, as in Figure 1 each Euclidean triangle has inner angles $\left(\varphi(f, e), \varphi\left(f^{\prime}, e\right), \pi-\right.$ $D(e))$. Then

$$
\begin{aligned}
\sum_{f \in F} \frac{1}{2} \Phi(f) & =\sum_{f \in F} \sum_{e<f} \varphi(f, e) \\
& =\sum_{e \in E, e<f, e<f^{\prime}}\left(\varphi(f, e)+\varphi\left(f^{\prime}, e\right)\right) \\
& =\sum_{e \in E} D(e) .
\end{aligned}
$$

Given a nonempty proper subset of $F$, i.e. $X \subset F, X \neq F$, the set $E(X)=\{e \in$ $E \mid e<f \in X\}$ can be decomposed as a union of two disjoint subsets $E(X)_{1} \cup E(X)_{2}$, where $E(X)_{1}=\left\{e \in E \mid e<f \in X, e<f^{\prime} \notin X\right\}$ and $E(X)_{2}=\{e \in E \mid e<f \in$ $\left.X, e<f^{\prime} \in X\right\}$. An edge in $E(X)_{1}$ is contained in exact one of the faces in $X$ while an edge in $E(X)_{2}$ is contained in two of the faces in $X$. Then

$$
\begin{aligned}
\sum_{f \in X} \frac{1}{2} \Phi(f) & =\sum_{f \in X} \sum_{e<f} \varphi(f, e) \\
& =\sum_{e \in E(X)_{1}, f \in X} \varphi(f, e)+\sum_{e \in E(X)_{2}}\left(\varphi(f, e)+\varphi\left(f^{\prime}, e\right)\right) \\
& =\sum_{e \in E(X)_{1}, f \in X} \varphi(f, e)+\sum_{e \in E(X)_{2}} D(e) \\
& <\sum_{e \in E(X)_{1}} D(e)+\sum_{e \in E(X)_{2}} D(e) \\
& =\sum_{e \in E(X)} D(e) .
\end{aligned}
$$

Next we claim the mapping $P: \Delta \rightarrow \delta$ is one to one. In fact, let $r=\left(r\left(f_{1}\right), \ldots, r\left(f_{n}\right)\right)$ and $\bar{r}=\left(\bar{r}\left(f_{1}\right), \ldots, \bar{r}\left(f_{n}\right)\right)$ be two distinct points in $\Delta$. Let $F_{0}=\left\{f_{i} \in F \mid r\left(f_{i}\right)>\right.$ $\left.\bar{r}\left(f_{i}\right)\right\}$. It is a nonempty proper subset. By the calculation above for a general subset $X$, we have

$$
\sum_{f \in F_{0}} \frac{1}{2} \Phi(f)=\sum_{e \in E\left(F_{0}\right)_{1}, f \in F_{0}} \varphi(f, e)+\sum_{e \in E\left(F_{0}\right)_{2}} D(e) .
$$

In a Euclidean triangle with inner angles $\left(\varphi(f, e), \varphi\left(f^{\prime}, e\right), \pi-D(e)\right)$, if the edge length $r(f)$ decreases and the edge length $r\left(f^{\prime}\right)$ increases or does not change, the angle $\varphi(f, e)$ increases. Thus if $e \in E\left(F_{0}\right)_{1}$ and $f \in F_{0}$, as a function of $r, \varphi(f, e)$ satisfies $\varphi(f, e)(r)<\varphi(f, e)(\bar{r})$. Therefore $\sum_{f \in F_{0}} \frac{1}{2} \Phi(f)(r)<\sum_{f \in F_{0}} \frac{1}{2} \Phi(f)(\bar{r})$. This shows $P: \Delta \rightarrow \delta$ is one to one.

Therefore $P: \Delta \rightarrow \delta$ is a smooth embedding. Thus $P(\Delta)$ is open in $\delta$. We will show $P(\Delta)$ is also closed in $\delta$. This will conclude that $P(\Delta)=\delta$ since $\delta$ is connected. To show the closeness we only need to show that $P$ sends the boundary of $\bar{\Delta}$ to the boundary of $\bar{\delta}$. More precisely, if there is a sequence of points $r^{(m)}$ in $\Delta$ so that $\lim _{m \rightarrow \infty} r^{(m)}=s=\left(s\left(f_{1}\right), \ldots, s\left(f_{n}\right)\right) \in \partial \bar{\Delta}$, then $\lim _{m \rightarrow \infty} P\left(r^{(m)}\right) \in \partial \bar{\delta}$, where we denote the boundary of a closed set $A$ by $\partial A$. Since $s \in \partial \bar{\Delta}, F_{1}=\left\{f_{i} \in F \mid s\left(f_{i}\right)=0\right\}$ 
is a nonempty proper subset. We have

$$
\sum_{f \in F_{1}} \frac{1}{2} \Phi(f)\left(r^{(m)}\right)=\sum_{e \in E\left(F_{1}\right)_{1}, f \in F_{1}} \varphi(f, e)\left(r^{(m)}\right)+\sum_{e \in E\left(F_{1}\right)_{2}} D(e) .
$$

If $e \in E\left(F_{1}\right)_{1}, e<f \in F_{1}$ and $e<f^{\prime} \notin F_{1}$, since $\lim _{m \rightarrow \infty}\left(r(f)\left(r^{(m)}\right), r\left(f^{\prime}\right)\left(r^{(m)}\right)\right)=$ $\left(s(f)=0, s\left(f^{\prime}\right)>0\right)$, we see that $\lim _{m \rightarrow \infty} \varphi(f, e)\left(r^{(m)}\right)=D(e)$. Hence

$$
\begin{aligned}
\lim _{m \rightarrow \infty} \sum_{f \in F_{1}} \frac{1}{2} \Phi(f)\left(r^{(m)}\right) & =\sum_{e \in E\left(F_{1}\right)_{1}} D(e)+\sum_{e \in E\left(F_{1}\right)_{2}} D(e) \\
& =\sum_{e \in E\left(F_{1}\right)} D(e) .
\end{aligned}
$$

By the definition of $\delta$, this shows that $\lim _{m \rightarrow \infty} P\left(r^{(m)}\right) \in \partial \bar{\delta}$.

\section{Continuity method for hyperbolic Circle PATtern}

Lemma 3.1. For a hyperbolic triangle with edge lengths $l, r_{1}, r_{2}$ and opposite angles $\theta, \varphi_{1}, \varphi_{2}$ respectively, when $\theta \in(0, \pi)$ is fixed, $\varphi_{1}, \varphi_{2}$ are functions of $r_{1}, r_{2}$.

(1) If $r_{1}$ decreases and $r_{2}$ increases or does not change, then $\varphi_{2}$ increases.

(2) If both of $r_{1}, r_{2}$ decrease, then $\varphi_{1}+\varphi_{2}$ increase.

(3) If $\left(r_{1}, r_{2}\right)$ converges to $(0, a)$, where $a \in(0, \infty)$, then $\varphi_{2}$ converges to $\pi-\theta$.

(4) If $\left(r_{1}, r_{2}\right)$ converges to $(0,0)$, then $\varphi_{1}+\varphi_{2}$ converges to $\pi-\theta$.

(5) If $\left(r_{1}, r_{2}\right)$ converges to $(\infty, b)$, where $b \in[0, \infty)$, then $\varphi_{2}$ converges to 0 .

(6) If $\left(r_{1}, r_{2}\right)$ converges to $(\infty, \infty)$, then $\varphi_{2}$ converges to to 0 .

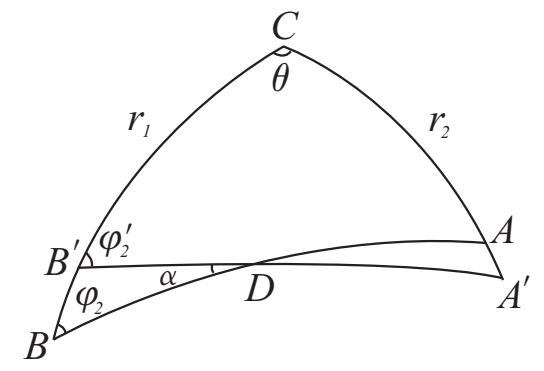

(a)

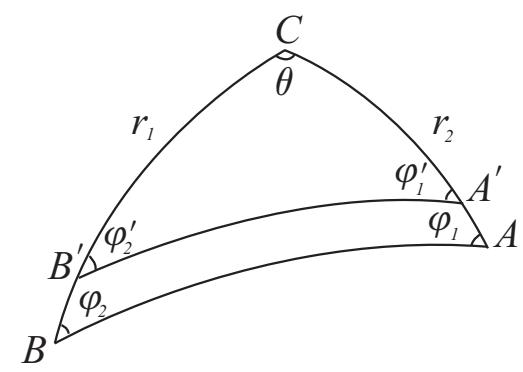

(b)

Figure 2. Angles change according to edge lengths

Proof. (1) As in the Figure2 (a), $\triangle_{A B C}$ is the hyperbolic triangle with edge lengths $r_{1}, r_{2}$ and angles $\varphi_{1}, \varphi_{2}$. If $r_{1}$ decreases and $r_{2}$ increases or does not change, we obtain another hyperbolic triangle $\triangle_{A^{\prime} B^{\prime} C}$ with angles $\varphi_{1}^{\prime}, \varphi_{2}^{\prime}$. Consider the hyperbolic triangle $\triangle_{B D B^{\prime}}$, we have $\alpha+\varphi_{2}+\pi-\varphi_{2}^{\prime}<\pi$. Thus $\varphi_{2}<\alpha+\varphi_{2}<\varphi_{2}^{\prime}$. This shows that $\varphi_{2}$ increases.

(2) As in the Figure2 (b), if both of $r_{1}, r_{2}$ decrease, we obtain another hyperbolic triangle $\triangle_{A^{\prime} B^{\prime} C}$ with angles $\varphi_{1}^{\prime}, \varphi_{2}^{\prime}$. Since the area of hyperbolic triangle $\triangle_{A^{\prime} B^{\prime} C}$ 
is less than that of $\triangle_{A B C}$, we see $\pi-\left(\theta+\varphi_{1}^{\prime}+\varphi_{2}^{\prime}\right)<\pi-\left(\theta+\varphi_{1}+\varphi_{2}\right)$. Thus $\varphi_{1}+\varphi_{2}<\varphi_{1}^{\prime}+\varphi_{2}^{\prime}$.

(3) If $\lim \left(r_{1}, r_{2}\right)=(0, a)$ with $a \in(0, \infty)$, by the cosine law, we see

$$
\begin{aligned}
\lim \cosh l & =\lim \left(\cosh r_{1} \cosh r_{2}-\cos \theta \sinh r_{1} \sinh r_{2}\right) \\
& =\cosh a .
\end{aligned}
$$

Thus $\lim l=a$. By the cosine law, we see

$$
\begin{aligned}
\lim \cos \varphi_{1} & =\lim \frac{-\cosh r_{1}+\cosh r_{2} \cosh l}{\sinh r_{2} \sinh l} \\
& =\frac{-1+\cosh ^{2} a}{\sinh ^{2} a} \\
& =1 .
\end{aligned}
$$

Thus $\lim \varphi_{1}=0$. Since the area of the hyperbolic triangle converges to 0 . We have $\lim \varphi_{2}=\pi-\theta$.

(4) If $\lim \left(r_{1}, r_{2}\right)=(0,0)$, the area of the hyperbolic triangle converges to 0 , then $\lim \left(\varphi_{1}+\varphi_{2}\right)=\pi-\theta$.

(5) If $\lim \left(r_{1}, r_{2}\right)=(\infty, b)$ with $b \in[0, \infty)$, by the triangle inequality $l>r_{1}-r_{2}$, then $\lim l=\infty$. Thus

$$
\begin{aligned}
\lim \cos \varphi_{2} & =\lim \frac{-\cosh r_{2}+\cosh r_{1} \cosh l}{\sinh r_{1} \sinh l} \\
& =\lim \frac{-\cosh r_{2}}{\sinh r_{1} \sinh l}+\lim \frac{\cosh r_{1}}{\sinh r_{1}} \frac{\cosh l}{\sinh l} \\
& =0+1 .
\end{aligned}
$$

Hence $\lim \varphi_{2}=0$.

(6) If $\lim \left(r_{1}, r_{2}\right)=(\infty, \infty)$, by the cosine law, we see

$\lim \cosh l=\lim \left(\cosh r_{1} \cosh r_{2}-\cos \theta \sinh r_{1} \sinh r_{2}\right)=\lim e^{r_{1}+r_{2}}(1-\cos \theta)=\infty$.

Thus $\lim l=\infty$. By the cosine law,

$$
\begin{aligned}
\lim \cos \varphi_{2} & =\lim \frac{-\cosh r_{2}+\cosh r_{1} \cosh l}{\sinh r_{1} \sinh l} \\
& =\lim \frac{-\cosh r_{2}}{\sinh r_{1} \sinh l}+1 \\
& =\lim \frac{-\cosh r_{2}}{\sinh r_{1} \cosh l}+1 \\
& =\lim \frac{-e^{r_{2}}}{e^{r_{1}} e^{r_{1}+r_{2}}(1-\cos \theta)}+1 \\
& =0+1 .
\end{aligned}
$$

Hence $\lim \varphi_{2}=0$.

Proof of Theorem 1.2. Assume the set of faces of the cell decomposition is $F=$ $\left\{f_{1}, \ldots, f_{n}\right\}$. Consider the space of radii $\Delta_{H}=\left\{\left(r\left(f_{1}\right), \ldots, r\left(f_{n}\right)\right)\right\}=\mathbb{R}_{>0}^{n}$. For a fixed intersection angle function $D: E \rightarrow(0, \pi)$, as in the Euclidean case there is a mapping $P_{H}: \Delta_{H} \rightarrow \mathbb{R}^{n}$ sending the radii $\left(r\left(f_{1}\right), \ldots, r\left(f_{n}\right)\right)$ to its cone angles $\Phi=\left(\Phi\left(f_{1}\right), \ldots, \Phi\left(f_{n}\right)\right)$. Now we introduce the polytope $\delta_{H}=\{\Phi \in$ $\mathbb{R}_{>0}^{n} \mid \Phi$ satisfies the condition (1.3) $\}$. We claim that $P_{H}\left(\Delta_{H}\right) \subset \delta_{H}$. 
In fact, for any nonempty subset $X$ of $F$, let's recall the decomposition $E(X)=$ $E(X)_{1} \cup E(X)_{2}$. Notice as in Figure1, in each hyperbolic triangle we have $\varphi(f, e)+$ $\varphi\left(f^{\prime}, e\right)<D(e)$. Thus

$$
\begin{aligned}
\sum_{f \in X} \frac{1}{2} \Phi(f) & =\sum_{f \in X} \sum_{e<f} \varphi(f, e) \\
& =\sum_{e \in E(X)_{1}, f \in X} \varphi(f, e)+\sum_{e \in E(X)_{2}}\left(\varphi(f, e)+\varphi\left(f^{\prime}, e\right)\right) \\
& <\sum_{e \in E(X)_{1}, f \in X} \varphi(f, e)+\sum_{e \in E(X)_{2}} D(e) \\
& <\sum_{e \in E(X)_{1}} D(e)+\sum_{e \in E(X)_{2}} D(e) \\
& =\sum_{e \in E(X)} D(e) .
\end{aligned}
$$

Next, we claim the mapping $P_{H}: \Delta_{H} \rightarrow \delta_{H}$ is one to one. In fact, let $r=$ $\left(r\left(f_{1}\right), \ldots, r\left(f_{n}\right)\right), \bar{r}=\left(\bar{r}\left(f_{1}\right), \ldots, \bar{r}\left(f_{n}\right)\right)$ be two distinct points in $\Delta_{H}$. Let $F_{0}=\left\{f_{i} \in\right.$ $\left.F \mid r\left(f_{i}\right)>\bar{r}\left(f_{i}\right)\right\}$ and $\bar{F}_{0}=\left\{f_{i} \in F \mid r\left(f_{i}\right)<\bar{r}\left(f_{i}\right)\right\}$. Since $r \neq r^{\prime}$, at least one of $F_{0}, \bar{F}_{0}$ is nonempty, say $F_{0}$ is nonempty. Now we have

$$
\sum_{f \in F_{0}} \frac{1}{2} \Phi(f)=\sum_{e \in E\left(F_{0}\right)_{1}, f \in F_{0}} \varphi(f, e)+\sum_{e \in E\left(F_{0}\right)_{2}}\left(\varphi(f, e)+\varphi\left(f^{\prime}, e\right)\right) .
$$

If $e \in E\left(F_{0}\right)_{1}, e<f \in F_{0}$ and $e<f^{\prime} \notin F_{0}$, then $r(f)$ decreases while $r\left(f^{\prime}\right)$ increases or does not change, by Lemma 3.1 $(1), \varphi(f, e)(r)<\varphi(f, e)(\bar{r})$. If $e \in E\left(F_{0}\right)_{2}$, then both of $r(f)$ and $r\left(f^{\prime}\right)$ decrease, by Lemma 3.1 (2), $\varphi(f, e)(r)+\varphi\left(f^{\prime}, e\right)(r)<$ $\varphi(f, e)(\bar{r})+\varphi\left(f^{\prime}, e\right)(\bar{r})$. Therefore $\sum_{f \in F_{0}} \frac{1}{2} \Phi(f)(r)<\sum_{f \in F_{0}} \frac{1}{2} \Phi(f)(\bar{r})$. This shows $P_{H}: \Delta_{H} \rightarrow \delta_{H}$ is one to one.

Therefore Therefore $P_{H}: \Delta_{H} \rightarrow \delta_{H}$ is a smooth embedding. Thus $P_{H}\left(\Delta_{H}\right)$ is open in $\delta_{H}$. We will show $P_{H}\left(\Delta_{H}\right)$ is also closed in $\delta_{H}$. This will conclude that $P_{H}\left(\Delta_{H}\right)=\delta_{H}$ since $\delta_{H}$ is connected. To show the closeness, we assume $r^{(m)}$ is a sequence of points in $\Delta_{H}$ such that $\lim _{m \rightarrow \infty} P_{H}\left(r^{(m)}\right)=t \in \delta_{H}$. By taking subsequence, we may assume $\lim _{m \rightarrow \infty} r^{(m)}=s \in[0, \infty]^{n}$. We only need to show $s \in(0, \infty)^{n}=\Delta_{H}$. This will finish the proof since $P_{H}(s)=t$.

Suppose otherwise that there is some face $f$ so that $s(f) \in\{0, \infty\}$, we will discuss the two cases.

Case 1 , if there is some face $f$ so that $\lim _{m \rightarrow \infty} r^{(m)}(f)=s(f)=\infty$, by Lemma 3.1(5) and (6), we have $\lim _{m \rightarrow \infty} \varphi(f, e)\left(r^{(m)}\right)=0$ for each $e<f$. Thus

$$
\begin{aligned}
t(f) & =\lim _{m \rightarrow \infty} \Phi(f)\left(r^{(m)}\right) \\
& =\lim _{m \rightarrow \infty} \sum_{e<f} \varphi(f, e)\left(r^{(m)}\right) \\
& =0 .
\end{aligned}
$$

This is a contradiction since we assume $t \in \delta_{H}$. 
Case 2, there is no face $f$ such that $s(f)=\infty$ but the subset $F_{1}=\left\{f_{i} \in\right.$ $\left.F \mid s\left(f_{i}\right)=0\right\}$ is nonempty. We have

$$
\sum_{f \in F_{1}} \frac{1}{2} \Phi(f)\left(r^{(m)}\right)=\sum_{e \in E\left(F_{1}\right)_{1}, f \in F_{1}} \varphi(f, e)\left(r^{(m)}\right)+\sum_{e \in E\left(F_{1}\right)_{2}}\left(\varphi(f, e)+\varphi\left(f^{\prime}, e\right)\right)\left(r^{(m)}\right) .
$$

If $e \in E\left(F_{1}\right)_{1}, e<f \in F_{1}$ and $e<f^{\prime} \notin F_{1}$, then $\lim _{m \rightarrow \infty}\left(r^{(m)}(f), r^{(m)}\left(f^{\prime}\right)\right)=$ $\left(0, s\left(f^{\prime}\right)>0\right)$, by Lemma $3.1(3), \lim _{m \rightarrow \infty} \varphi(f, e)\left(r^{(m)}\right)=D(e)$. If $e \in E\left(F_{1}\right)_{2}$, then $\lim _{m \rightarrow \infty}\left(r^{(m)}(f), r^{(m)}\left(f^{\prime}\right)\right)=(0,0)$, by Lemma $3.1(4), \lim _{m \rightarrow \infty}(\varphi(f, e)+$ $\left.\varphi\left(f^{\prime}, e\right)\right)\left(r^{(m)}\right)=D(e)$. Hence

$$
\begin{aligned}
\lim _{m \rightarrow \infty} \sum_{f \in F_{1}} \frac{1}{2} \Phi(f)\left(r^{(m)}\right) & =\sum_{e \in E\left(F_{1}\right)_{1}} D(e)+\sum_{e \in E\left(F_{1}\right)_{2}} D(e) \\
& =\sum_{e \in E\left(F_{1}\right)} D(e) .
\end{aligned}
$$

This is a contradiction since we assume $t \in \delta_{H}$.

\section{VARIATIONAL PRINCIPLE FOR HYPERBOLIC CIRClE PATTERN}

4.1. Angle structure. An angle structure for a hyperbolic circle pattern with an intersection angle function $D: E \rightarrow(0, \pi)$ and a cone angle function $\Phi: F \rightarrow(0, \infty)$ is a function $\varphi: F \times E \rightarrow(0, \pi)$, such that $\varphi(f, e)+\varphi\left(f^{\prime}, e\right)<D(e)$ for each two faces $f, f^{\prime}$ sharing an edge $e$ and $\sum_{e<f} \varphi(f, e)=\frac{1}{2} \Phi(f)$ for each face $f$. Denote the space of all angle structures by $\mathcal{A}$. In this section we will use the duality theorem in the linear programming. For a statement and a proof of the theorem, see for example Kolman and Beck [5].

Lemma 4.1. Given a cellular decomposed surface $\Sigma$, an intersection angle function $D: E \rightarrow(0, \pi)$ and a cone angle function $\Phi: F \rightarrow(0, \infty)$, the closure of $\mathcal{A}$ is nonempty if and only if

$$
\sum_{f \in X} \frac{1}{2} \Phi(f) \leq \sum_{e \in E(X)} D(e)
$$

for any $X \subseteq F$.

Proof. Consider the linear programming problem $(P)$ : minimize the objective function $z=0$, subject to the constraints

$$
\left\{\begin{array}{l}
\varphi(f, e)+\varphi\left(f^{\prime}, e\right) \leq D(e) \\
\sum_{e<f} \varphi(f, e)=\frac{1}{2} \Phi(f) \\
\varphi(f, e) \geq 0
\end{array}\right.
$$

The closure of $\mathcal{A}$ is exactly the set of solutions of above inequalities.

The dual problem $\left(P^{*}\right)$ has variables $\left(\ldots, y_{f}, \ldots, y_{e}, \ldots\right)$ indexed by $F \cup E$. The dual problem $\left(P^{*}\right)$ is to maximize the objective function $z=\sum_{f \in F} \frac{1}{2} \Phi(f) y_{f}+$ $\sum_{e \in E} D(e) y_{e}$, subject to the constraints

$$
\left\{\begin{array}{l}
y_{e} \leq 0 \\
y_{f}+y_{e} \leq 0 \text { whenever } e<f
\end{array}\right.
$$

Now by the definition of $\mathcal{A}$ and the duality theorem in the linear programming, the following statements are equivalent.

(i) The closure of $\mathcal{A}$ is nonempty. 
(ii) There exists a feasible solution of the problem $(P)$.

(iii) The objective function of the problem $\left(P^{*}\right)$ is non-positive.

Hence to prove this lemma we only need to show the condition (4.1) is equivalent to the statement (iii).

To show the condition (4.1) is necessary, we assume that the objective function of $\left(P^{*}\right)$ is non-positive. For any $X \subseteq F$, let

$$
y_{f}=\left\{\begin{array}{ll}
1 & \text { if } f \in X \\
0 & \text { if } f \notin X
\end{array} \text { and } y_{e}=\left\{\begin{array}{cl}
-1 & \text { if } e \in E(X) \\
0 & \text { if } e \notin E(X)
\end{array}\right.\right.
$$

It is easy to check $\left(y_{f}, y_{e}\right)$ is a feasible solution of $\left(P^{*}\right)$. Thus $0 \geq z\left(y_{f}, y_{e}\right)=$ $\sum_{f \in X} \frac{1}{2} \Phi(f) y_{f}+\sum_{e \in E(X)} D(e) y_{e}=\sum_{f \in X} \frac{1}{2} \Phi(f)-\sum_{e \in E(X)} D(e)$. This gives the condition (4.1).

To show the condition (4.1) is sufficient, take an arbitrary feasible solution of $\left(P^{*}\right):\left(y_{f}, y_{e}\right)$. Since we can replace the negative $y_{f}$ 's by 0 's such that the objective function does not decrease, we can assume all $y_{f} \geq 0$. Let $X=\left\{f \in F \mid y_{f}>0\right\}$, and $a$ be the minimal element in the set $\left\{y_{f}, f \in X\right\}$. Thus $a>0$. Then we define

$$
y_{f}^{(1)}=\left\{\begin{array}{ll}
y_{f}-a & \text { if } f \in X \\
y_{f}=0 & \text { if } f \notin X
\end{array} \text { and } y_{e}^{(1)}=\left\{\begin{array}{cl}
y_{e}+a & \text { if } e \in E(X) \\
y_{e} & \text { if } e \notin E(X)
\end{array}\right.\right.
$$

We can check $\left(y_{f}^{(1)}, y_{e}^{(1)}\right)$ is still a feasible solution of $\left(P^{*}\right)$. In fact if $e \notin E(X)$, then $y_{e}^{(1)}=y_{e} \leq 0$. If $e \in E(X)$, there exists $f^{\prime} \in X$ such that $e<f^{\prime}$. Then $y_{e}^{(1)}=y_{e}+a \leq y_{e}+y_{f^{\prime}} \leq 0$, since $a$ is minimal and by assumption $\left(y_{f^{\prime}}, y_{e}\right)$ is feasible. Next we consider $y_{f}^{(1)}+y_{e}^{(1)}$. If $f \in X, y_{f}^{(1)}+y_{e}^{(1)}=y_{f}+y_{e} \leq 0$ or $y_{f}^{(1)}+y_{e}^{(1)}=y_{f}-a+y_{e}<0$. If $f \notin X, y_{f}^{(1)}+y_{e}^{(1)}=y_{e}^{(1)} \leq 0$. Hence $\left(y_{f}^{(1)}, y_{e}^{(1)}\right)$ is feasible.

Now $z\left(y_{f}^{(1)}, y_{e}^{(1)}\right)=z\left(y_{f}, y_{e}\right)+a\left(\sum_{e \in E(X)} D(e)-\sum_{f \in X} \frac{1}{2} \Phi(f)\right) \geq z\left(y_{f}, y_{e}\right)$. due to the condition (4.1). Notice that the number of 0 's in $\left\{y_{f}^{(1)}\right\}$ is more than that in $\left\{y_{f}\right\}$. By the same procedure, we obtain a sequence of feasible solutions $\left(y_{f}^{(i)}, y_{e}^{(i)}\right)$. After finite steps, it ends at a feasible solution $\left(y_{f}^{(n)}=0, y_{e}^{(n)} \leq 0\right)$. Thus $z\left(y_{f}, y_{e}\right) \leq z\left(y_{f}^{(1)}, y_{e}^{(1)}\right) \leq \ldots \leq z\left(y_{f}^{(n)}, y_{e}^{(n)}\right)=\sum_{e \in E} D(e) y_{e}^{(n)} \leq 0$. This shows that the objective function of $\left(P^{*}\right)$ is non-positive.

Theorem 4.2 (Bobenko-Springborn [1] Proposition 4). Given a cellular decomposed surface $\Sigma$, an intersection angle function $D: E \rightarrow(0, \pi)$ and a cone angle function $\Phi: F \rightarrow(0, \infty), \mathcal{A}$ is nonempty if and only if

$$
\sum_{f \in X} \frac{1}{2} \Phi(f)<\sum_{e \in E(X)} D(e)
$$

for any $X \subseteq F$.

Proof. Let $\varphi(f, e)=a(f, e)+\varepsilon$, where $a(f, e) \geq 0$ and $\varepsilon \geq 0$. Consider the linear programming problem $(P)$ with variables $\{\ldots, a(f, e), \ldots \varepsilon\}$ : minimize the objective 
function $z=-\varepsilon$, subject to the constraints

$$
\left\{\begin{array}{l}
a(f, e)+a\left(f^{\prime}, e\right)+2 \varepsilon \leq D(e) \\
\sum_{e<f} a(f, e)+n_{f} \varepsilon=\frac{1}{2} \Phi(f) \\
a(f, e) \geq 0 \\
\varepsilon \geq 0
\end{array}\right.
$$

where $n_{f}$ is the number of edges of the face $f$.

The dual problem $\left(P^{*}\right)$ has variables $\left(\ldots, y_{f}, \ldots, y_{e}, \ldots\right)$ indexed by $F \cup E$. The dual problem $\left(P^{*}\right)$ is to maximize the objective function $z=\sum_{f \in F} \frac{1}{2} \Phi(f) y_{f}+$ $\sum_{e \in E} D(e) y_{e}$, subject to the constraints

$$
\left\{\begin{array}{l}
y_{e} \leq 0 \\
y_{f}+y_{e} \leq 0 \quad \text { whenever } e<f \\
\sum_{f \in F} n_{f} y_{f}+2 \sum_{e \in E} y_{e} \leq-1
\end{array}\right.
$$

Now by the definition of $\mathcal{A}$ and the duality theorem in the linear programming, the following statements are equivalent.

(i) The set $\mathcal{A}$ is nonempty.

(ii) There exists a feasible solution of the problem $(P)$ such that $a(f, e) \geq 0, \varepsilon>$ 0 .

(iii) The minimal value of the objective function of the problem $(P)$ is negative.

(iv) The objective function of the problem $\left(P^{*}\right)$ is negative.

Hence to prove this lemma we only need to show the condition (4.2) is equivalent to the statement (iv).

To show the condition (4.2) is necessary, if $X=F$, then

$$
\begin{aligned}
\sum_{f \in F} \frac{1}{2} \Phi(f) & =\sum_{f \in F} \sum_{e<f} \varphi(f, e) \\
& =\sum_{e \in E}\left(\varphi(f, e)+\varphi\left(f^{\prime}, e\right)\right) \\
& <\sum_{e \in E} D(e) .
\end{aligned}
$$

For any $X \subset F, X \neq F$ let

$$
y_{f}=\left\{\begin{array}{ll}
1 & \text { if } f \in X \\
0 & \text { if } f \notin X
\end{array} \text { and } y_{e}=\left\{\begin{array}{cl}
-1 & \text { if } e \in E(X) \\
0 & \text { if } e \notin E(X)
\end{array}\right.\right.
$$

To show $\left(y_{f}, y_{e}\right)$ is a feasible solution of $\left(P^{*}\right)$ we only need to check it satisfies $\sum_{f \in F} n_{f} y_{f}+2 \sum_{e \in E} y_{e} \leq-1$. This is equivalent to $\sum_{f \in X} n_{f}-2 \sum_{e \in E(X)} \leq-1$. It is true since $X \neq F$ implies that $2|E(X)|>\sum_{f \in X} n_{f}$ or $2|E(X)| \geq \sum_{f \in X} n_{f}+$ 1. Since the objective function is negative, we have $0>z\left(y_{f}, y_{e}\right)$ which implies $\sum_{f \in X} \frac{1}{2} \Phi(f)<\sum_{e \in E} D(e)$. This gives the condition (4.2).

To show the condition (4.2) is sufficient, by the proof of Lemma 4.1 we know the objective function of $\left(P^{*}\right)$ is $\leq 0$ under the condition (4.2) which is stronger than the condition (4.1). We try to show it can not be 0. Otherwise, let $\left(y_{f}, y_{e}\right)$ be a feasible solution satisfying $z\left(y_{f}, y_{e}\right)=0$. We obtain a new feasible solution $\left(y_{f}^{\prime}, y_{e}\right)$ of $\left(P^{*}\right)$ by replacing the negative $y_{f}$ 's by 0's. Since the objective function does not decrease, $0=z\left(y_{f}, y_{e}\right) \leq z\left(y_{f}^{\prime}, y_{e}\right) \leq 0$. If there is some face $f$ such that $y_{f}^{\prime}>0$, we can construct another feasible solution $\left(y_{f}^{(1)}, y_{e}^{(1)}\right)$ as in the proof of Lemma 4.1 
so that $z\left(y_{f}^{(1)}, y_{e}^{(1)}\right)>z\left(y_{f}^{\prime}, y_{e}\right)=0$. This is impossible since the objective function $z \leq 0$.

Hence $y_{f}^{\prime}=0$ for each $f$. Therefore $0=z\left(y_{f}^{\prime}, y_{e}\right)=\sum_{e \in E} D(e) y_{e}$. Since $y_{e} \leq 0$ for each $e$, we see that $y_{e}=0$ for each $e$. But $\left(y_{f}^{\prime}=0, y_{e}=0\right)$ does not satisfy the condition $\sum_{f \in F} n_{f} y_{f}+2 \sum_{e \in E} y_{e} \leq-1$. This contradiction implies that the objective function of $\left(P^{*}\right)$ can not be 0 .

4.2. The derivative cosine law. To prove Theorem 1.2 we will define an energy function on $\mathcal{A}$ the space of angle structures. First, we will construct a function for one triangle.

The following lemma is essentially obtained in Luo [7]. For the convenience of readers, we include a simple proof here. We use

$$
\left[\begin{array}{c}
a_{1} \\
\vdots \\
a_{n}
\end{array}\right]
$$

to denote the diagonal matrix with diagonal entries $\left(a_{1}, \ldots, a_{n}\right)$.

Lemma 4.3 (The derivative cosine law). For a hyperbolic triangle with three edges $l_{1}, l_{2}, l_{3}$ and opposite angles $\theta_{1}, \theta_{2}, \theta_{3},\{i, j, k\}=\{1,2,3\}$, the differentials of $l^{\prime} s$ and $\theta^{\prime}$ s satisfy

$$
\left(\begin{array}{l}
d l_{1} \\
d l_{2} \\
d l_{3}
\end{array}\right)=\frac{-1}{\sinh l_{i} \sinh l_{j} \sin \theta_{k}}\left[\begin{array}{c}
\sinh l_{1} \\
\sinh l_{2} \\
\sinh l_{3}
\end{array}\right]\left(\begin{array}{ccc}
1 & \cosh l_{3} & \cosh l_{2} \\
\cosh l_{3} & 1 & \cosh l_{1} \\
\cosh l_{2} & \cosh l_{1} & 1
\end{array}\right)\left(\begin{array}{l}
d \theta_{1} \\
d \theta_{2} \\
d \theta_{3}
\end{array}\right) .
$$

Proof. By differentiating the cosine law of hyperbolic triangles

$$
\cosh l_{i} \sin \theta_{j} \sin \theta_{k}=\cos \theta_{i}+\cos \theta_{j} \cos \theta_{k},
$$

we have

$$
\sinh l_{i} \sin \theta_{j} \sin \theta_{k} d l_{i}
$$

$=-\sin \theta_{i} d \theta_{i}-\left(\sin \theta_{j} \cos \theta_{k}+\cosh l_{i} \cos \theta_{j} \sin \theta_{k}\right) d \theta_{j}-\left(\cos \theta_{j} \sin \theta_{k}+\cosh l_{i} \sin \theta_{j} \cos \theta_{k}\right) d \theta_{k}$.

After replacing $\cosh l_{i}$ by using the $\operatorname{cosine~law~}$

$$
\cosh l_{i}=\frac{\cos \theta_{i}+\cos \theta_{j} \cos \theta_{k}}{\sin \theta_{j} \sin \theta_{k}}
$$

and simplifying we get

$$
\begin{aligned}
\sinh l_{i} \sin \theta_{j} \sin \theta_{k} d l_{i} & =-\sin \theta_{i} d \theta_{i}-\frac{\cos \theta_{i} \cos \theta_{j}+\cos \theta_{k}}{\sin \theta_{j}} d \theta_{j}-\frac{\cos \theta_{i} \cos \theta_{k}+\cos \theta_{j}}{\sin \theta_{k}} d \theta_{k} \\
& =-\sin \theta_{i}\left(d \theta_{i}+\cosh l_{k} d \theta_{j}+\cosh l_{j} d \theta_{k}\right) .
\end{aligned}
$$

The matrix is derived from the sine law:

$$
\frac{\sin \theta_{i}}{\sinh l_{i}}=\frac{\sin \theta_{j}}{\sinh l_{j}}, \text { for any } i, j \in\{1,2,3\} \text {. }
$$

Let the domain $T=\left\{\left(\varphi_{1}, \varphi_{2}\right) \mid \varphi_{1}>0, \varphi_{2}>0, \varphi_{1}+\varphi_{2}<\pi-\theta\right\}$ be the space of angles $\left(\varphi_{1}, \varphi_{2}\right)$ in a hyperbolic triangle with the third angle $\theta$ fixed. 
REN GUO

Lemma 4.4. For a hyperbolic triangle with three edges $r_{1}, r_{2}, l$ and opposite angles $\varphi_{1}, \varphi_{2}, \theta$, when $\theta \in(0, \pi)$ is fixed, let $w=\ln \tanh \frac{r_{1}}{2} d \varphi_{2}+\ln \tanh \frac{r_{2}}{2} d \varphi_{1}$. Then the function $v\left(\varphi_{1}, \varphi_{2}\right)=\int_{(0,0)}^{\left(\varphi_{1}, \varphi_{2}\right)} w$ is well defined and strictly concave down in the domain $T$.

Proof. If $\theta \in(0, \pi)$ is fixed, then $d \theta=0$, by Lemma 4.3, we have

$$
\begin{aligned}
\left(\begin{array}{l}
d r_{1} \\
d r_{2}
\end{array}\right) & =\frac{-1}{\sinh r_{1} \sinh r_{2} \sin \theta}\left[\begin{array}{l}
\sinh r_{1} \\
\sinh r_{2}
\end{array}\right]\left(\begin{array}{cc}
1 & \cosh l \\
\cosh l & 1
\end{array}\right)\left(\begin{array}{c}
d \varphi_{1} \\
d \varphi_{2}
\end{array}\right) \\
& =\frac{-1}{\sinh r_{1} \sinh r_{2} \sin \theta}\left[\begin{array}{c}
\sinh r_{1} \\
\sinh r_{2}
\end{array}\right]\left(\begin{array}{cc}
\cosh l & 1 \\
1 & \cosh l
\end{array}\right)\left(\begin{array}{c}
d \varphi_{2} \\
d \varphi_{1}
\end{array}\right) .
\end{aligned}
$$

Then

$$
\begin{aligned}
\left(\begin{array}{l}
d \ln \tanh \frac{r_{1}}{2} \\
d \ln \tanh \frac{r_{2}}{2}
\end{array}\right) & =\left[\begin{array}{l}
\sinh ^{-1} r_{1} \\
\sinh ^{-1} r_{2}
\end{array}\right]\left(\begin{array}{l}
d r_{1} \\
d r_{2}
\end{array}\right) \\
& =\frac{-1}{\sinh r_{1} \sinh r_{2} \sin \theta}\left(\begin{array}{cc}
\cosh l & 1 \\
1 & \cosh l
\end{array}\right)\left(\begin{array}{c}
d \varphi_{2} \\
d \varphi_{1}
\end{array}\right) .
\end{aligned}
$$

We denote it to be

$$
\left(\begin{array}{c}
d \ln \tanh \frac{r_{1}}{2} \\
d \ln \tanh \frac{r_{2}}{2}
\end{array}\right)=M\left(\begin{array}{c}
d \varphi_{2} \\
d \varphi_{1}
\end{array}\right)
$$

where $M$ is symmetric and negative definite. Hence $w=\ln \tanh \frac{r_{1}}{2} d \varphi_{2}+\ln \tanh \frac{r_{2}}{2} d \varphi_{1}$ is a closed $1-$ form in the domain $T$. Therefore the function $v\left(\varphi_{1}, \varphi_{2}\right)$ is well defined. Since the Hessian matrix of $v\left(\varphi_{1}, \varphi_{2}\right)$ is exactly the matrix $M$ which is negative definite, $v\left(\varphi_{1}, \varphi_{2}\right)$ is strictly concave down in $T$.

4.3. Boundary behavior. In this section we will investigate the behavior of the function $v\left(\varphi_{1}, \varphi_{2}\right)$ on the boundary of the closure of the domain $T$.

Lemma 4.5. The function $v\left(\varphi_{1}, \varphi_{2}\right)$ can be continuously extended to the boundary of the closure of the domain $T$.

Proof. By the cosine law

$$
\begin{aligned}
\left(\tanh \frac{r_{1}}{2}\right)^{2} & =\frac{\cosh r_{1}-1}{\cosh r_{1}+1} \\
& =\frac{\cos \varphi_{1}+\cos \left(\varphi_{2}+\theta\right)}{\cos \varphi_{1}-\cos \left(\varphi_{2}+\theta\right)} \\
& =\frac{\sin \frac{\pi-\theta-\varphi_{1}-\varphi_{2}}{2} \sin \frac{\pi+\theta-\varphi_{1}+\varphi_{2}}{2}}{\sin \frac{\pi+\theta-\varphi_{1}-\varphi_{2}}{2} \sin \frac{\pi-\theta-\varphi_{1}+\varphi_{2}}{2}} .
\end{aligned}
$$

By interchange the index 1,2 , we obtain the similar formula.

$$
\begin{aligned}
\left(\tanh \frac{r_{2}}{2}\right)^{2} & =\frac{\sin \frac{\pi-\theta-\varphi_{1}-\varphi_{2}}{2} \sin \frac{\pi+\theta-\varphi_{2}+\varphi_{1}}{2}}{\sin \frac{\pi+\theta-\varphi_{1}-\varphi_{2}}{2} \sin \frac{\pi-\theta-\varphi_{2}+\varphi_{1}}{2}} \\
& =\frac{\sin \frac{\pi-\theta-\varphi_{1}-\varphi_{2}}{2} \sin \frac{\pi-\theta-\varphi_{1}+\varphi_{2}}{2}}{\sin \frac{\pi+\theta-\varphi_{1}-\varphi_{2}}{2} \sin \frac{\pi+\theta-\varphi_{1}+\varphi_{2}}{2}}
\end{aligned}
$$

due to $\frac{\pi+\theta-\varphi_{2}+\varphi_{1}}{2}+\frac{\pi-\theta-\varphi_{1}+\varphi_{2}}{2}=\pi$. 
Thus

$$
\begin{aligned}
w & =\frac{1}{2}\left(\ln \sin \frac{\pi-\theta-\varphi_{1}-\varphi_{2}}{2}-\ln \sin \frac{\pi+\theta-\varphi_{1}-\varphi_{2}}{2}\right)\left(d \varphi_{2}+d \varphi_{1}\right) \\
& +\frac{1}{2}\left(\ln \sin \frac{\pi+\theta-\varphi_{1}+\varphi_{2}}{2}-\ln \sin \frac{\pi-\theta-\varphi_{1}+\varphi_{2}}{2}\right)\left(d \varphi_{2}-d \varphi_{1}\right) .
\end{aligned}
$$

Hence

$$
\begin{aligned}
v\left(\varphi_{1}, \varphi_{2}\right) & =\Lambda\left(\frac{\pi-\theta-\varphi_{1}-\varphi_{2}}{2}\right)+\Lambda\left(\frac{\pi-\theta+\varphi_{1}+\varphi_{2}}{2}\right) \\
& +\Lambda\left(\frac{\pi-\theta+\varphi_{1}-\varphi_{2}}{2}\right)+\Lambda\left(\frac{\pi-\theta-\varphi_{1}+\varphi_{2}}{2}\right)-4 \Lambda\left(\frac{\pi-\theta}{2}\right),
\end{aligned}
$$

where $\Lambda(x)=-\int_{0}^{x} \ln |2 \sin t| d t$ is the Lobachevsky function. By the property of Lobachevsky function, function $v\left(\varphi_{1}, \varphi_{2}\right)$ can be continuously extended to the boundary of the domain $T$.

The closure of the domain $T=\left\{\left(\varphi_{1}, \varphi_{2}\right) \mid \varphi_{1}>0, \varphi_{2}>0, \varphi_{1}+\varphi_{2}<\pi-\theta\right\}$ is a triangle with vertexes $O=(0,0), V_{1}=(\pi-\theta, 0), V_{2}=(0, \pi-\theta)$. If $\left(\varphi_{1}, \varphi_{2}\right)$ is in the line segment of $V_{1} V_{2}$, the triangle with angles $\left(\varphi_{1}, \varphi_{2}, \theta\right)$ is a Euclidean or degenerated Euclidean triangle. Otherwise the triangle with angles $\left(\varphi_{1}, \varphi_{2}, \theta\right)$ is a hyperbolic or degenerated hyperbolic triangle. Let $\left(\varphi_{1}(t), \varphi_{2}(t)\right)$ be a line segment, where, for $i=1,2$ and $0 \leq t \leq 1$,

$$
\varphi_{i}(t)=(1-t) \varphi_{i}(0)+t \varphi_{i}(1),
$$

such that $\left(\varphi_{1}(1), \varphi_{2}(1)\right) \in T$ and $\left(\varphi_{1}(0), \varphi_{2}(0)\right)$ is in the closure of $T$.

Lemma 4.6. Let $v(t)=v\left(\varphi_{1}(t), \varphi_{2}(t)\right)$.

(1) If $\left(\varphi_{1}(0), \varphi_{2}(0)\right)$ is in the line segment of $V_{1} V_{2}$, then $\lim _{t \rightarrow 0} v^{\prime}(t)=\infty$.

(2) Otherwise, $\lim _{t \rightarrow 0} v^{\prime}(t)$ exists and is a finite number.

Proof. We have

$$
v^{\prime}(t)=\frac{d}{d t} v\left(\varphi_{1}(t), \varphi_{2}(t)\right)=\varphi_{2}^{\prime}(t) \ln \tanh \frac{r_{1}(t)}{2}+\varphi_{1}^{\prime}(t) \ln \tanh \frac{r_{2}(t)}{2},
$$

where $r_{1}(t)$ and $r_{2}(t)$ are the edge lengths of the hyperbolic triangle with angles $\left(\varphi_{1}(t), \varphi_{2}(t), \theta\right)$.

Case 1, if $\left(\varphi_{1}(0), \varphi_{2}(0)\right) \in T$, in the hyperbolic triangle with angles $\left(\varphi_{1}(0), \varphi_{2}(0), \theta\right)$, the edge lengths $r_{1}(0), r_{2}(0)$ are finite. Hence $\lim _{t \rightarrow 0} v^{\prime}(t)$ exists and is a finite number.

Case 2, if $\left(\varphi_{1}(0), \varphi_{2}(0)\right)$ is in the interior of the segment $O V_{1}$ (similar for $\left.O V_{2}\right)$, in the hyperbolic triangle with angles $\left(\varphi_{1}(0), \varphi_{2}(0)=0, \theta\right)$, the edge lengths $r_{1}(0)$ is infinite and the edge length $r_{2}(0)$ is finite. Hence $\lim _{t \rightarrow 0} v^{\prime}(t)=\varphi_{1}^{\prime}(0) \ln \tanh \frac{r_{2}(0)}{2}=$ $\left(\varphi_{1}(1)-\varphi_{1}(0)\right) \ln \tanh \frac{r_{2}(0)}{2}$ is a finite number.

Case 3 , if $\left(\varphi_{1}(0), \varphi_{2}(0)\right)=O$, in the hyperbolic triangle with angles $\left(\varphi_{1}(0)=\right.$ $\left.0, \varphi_{2}(0)=0, \theta\right)$, the two edge lengths $r_{1}(0), r_{2}(0)$ are infinite. Hence $\lim _{t \rightarrow 0} v^{\prime}(t)=$ 0 .

If $\left(\varphi_{1}(0), \varphi_{2}(0)\right)$ is in the segment of $V_{1} V_{2},\left(\varphi_{1}(0), \varphi_{2}(0), \theta\right)$ are the angles of a Euclidean or degenerated Euclidean triangle. Thus the two edge lengths $r_{1}(0), r_{2}(0)$ turn to zero. 
By the computation in Lemma 4.5, we have

$$
\begin{aligned}
v^{\prime}(t) & =\frac{1}{2}\left(\varphi_{2}^{\prime}(t)+\varphi_{1}^{\prime}(t)\right)\left(\ln \sin \frac{\pi-\theta-\varphi_{1}-\varphi_{2}}{2}-\ln \sin \frac{\pi+\theta-\varphi_{1}-\varphi_{2}}{2}\right) \\
& +\frac{1}{2}\left(\varphi_{2}^{\prime}(t)-\varphi_{1}^{\prime}(t)\right)\left(\ln \sin \frac{\pi+\theta-\varphi_{1}+\varphi_{2}}{2}-\ln \sin \frac{\pi-\theta-\varphi_{1}+\varphi_{2}}{2}\right) .
\end{aligned}
$$

Case 4 , if $\left(\varphi_{1}(0), \varphi_{2}(0)\right)$ is in the interior of the segment $V_{1} V_{2}$, then $\varphi_{1}(0)+\varphi_{2}(0)+$ $\theta=\pi$ and $\varphi_{1}(0)>0, \varphi_{2}(0)>0$. Thus $\lim _{t \rightarrow 0} v^{\prime}(t)=\frac{1}{2}\left(\varphi_{2}^{\prime}(0)+\varphi_{1}^{\prime}(0)\right) \ln \sin 0+$ finite terms.

Since $\varphi_{1}^{\prime}(0)+\varphi_{2}^{\prime}(0)=\left(\varphi_{1}(1)+\varphi_{2}(1)\right)-\left(\varphi_{1}(0)+\varphi_{2}(0)\right)<(\pi-\theta)-(\pi-\theta)=0$, then $\lim _{t \rightarrow 0} v^{\prime}(t)=\infty$.

Case 5, if $\left(\varphi_{1}(0), \varphi_{2}(0)\right)=V_{1}\left(\right.$ similar for $\left.V_{2}\right)$, then $\varphi_{1}(0)=\pi-\theta, \varphi_{2}(0)=0$. Thus $\lim _{t \rightarrow 0} v^{\prime}(t)=\frac{1}{2}\left(\varphi_{2}^{\prime}(0)+\varphi_{1}^{\prime}(0)\right)(\ln \sin 0-\ln \sin \theta)+\frac{1}{2}\left(\varphi_{2}^{\prime}(0)-\varphi_{1}^{\prime}(0)\right)(\ln \sin \theta-$ $\ln \sin 0)=\varphi_{1}^{\prime}(0)(\ln \sin 0-\ln \sin \theta)=\left(\varphi_{1}(1)-\varphi_{1}(0)\right)(\ln \sin 0-\ln \sin \theta)$. Since $\varphi_{1}(0)=$ $\pi-\theta>\varphi_{1}(1)$, then $\lim _{t \rightarrow 0} v^{\prime}(t)=\infty$.

4.4. Proof of Theorem 1.2. Given a cellular decomposed surface $\Sigma$, an intersection angle function $D: E \rightarrow(0, \pi)$ and a cone angle function $\Phi: F \rightarrow(0, \infty)$, if there exists a hyperbolic circle pattern, for each edge $e$ there is a quadrilateral which is a union of two congruent hyperbolic triangles. The angles of these triangles define an angle structure. By Theorem 4.2, the condition (1.3) holds.

On the other hand, if the condition (1.3) holds, by Theorem 4.2, $\mathcal{A}$ the space of all angle structures is nonempty. Given an angle structure $\varphi \in \mathcal{A}$, for each edge $e$ shared by face $f$ and $f^{\prime}$, there is a topological quadrilateral. We realize the quadrilateral as a union of two congruent hyperbolic triangles with angles $\left(\varphi(f, e), \varphi\left(f^{\prime}, e\right), \pi-D(e)\right)$. Let $r(f, e)$ and $r\left(f^{\prime}, e\right)$ be the edge lengths of one of the two triangles. Since the two triangles are always congruent, we only need to take care one of them.

For each hyperbolic triangle we have defined a function $v$. Summation of the function $v$ over "half" of all of triangles produces an energy function $\mathcal{E}: \mathcal{A} \rightarrow \mathbb{R}$. In fact for $\varphi \in \mathcal{A}$, let

$$
\begin{aligned}
\mathcal{E}(\varphi) & =\sum_{e \in E} v\left(\varphi(f, e), \varphi\left(f^{\prime}, e\right)\right) \\
& =\sum_{e \in E} \int_{(0,0)}^{\left(\varphi(f, e), \varphi\left(f^{\prime}, e\right)\right)}\left(\ln \tanh \frac{r(f, e)}{2} d \varphi(f, e)+\ln \tanh \frac{r\left(f^{\prime}, e\right)}{2} d \varphi\left(f^{\prime}, e\right)\right),
\end{aligned}
$$

where faces $f, f^{\prime}$ share the edge $e, r(f, e)$ and $r\left(f^{\prime}, e\right)$ are the edge lengths of the hyperbolic triangle with angles $\left(\varphi(f, e), \varphi\left(f^{\prime}, e\right), \pi-D(e)\right)$. By Lemma 4.4 and Lemma 4.5. the function $\mathcal{E}$ is strictly concave down in $\mathcal{A}$ and can be continuously extended to the boundary of the closure of $\mathcal{A}$. Since the closure of $\mathcal{A}$ is compact, $\mathcal{E}$ has the unique maximum point $\varphi_{0}$ in closure of $\mathcal{A}$.

First we claim that under the angle structure $\varphi_{0}$, there is no Euclidean or degenerated Euclidean triangles. Otherwise, choose any point $\varphi_{1} \in \mathcal{A}$ and join $\varphi_{0}$ and $\varphi_{1}$ by a line segment $\varphi_{t}=(1-t) \varphi_{0}+t \varphi_{1}$. Let $E=I \cup J$, where each edge in $I$ corresponds to a hyperbolic or degenerate hyperbolic triangle while each edge in $J$ corresponds to a Euclidean or degenerated Euclidean triangle. Then

$$
\mathcal{E}\left(\varphi_{t}\right)=\sum_{e \in I} v\left(\varphi_{t}(f, e), \varphi_{t}\left(f^{\prime}, e\right)\right)+\sum_{e \in J} v\left(\varphi_{t}(f, e), \varphi_{t}\left(f^{\prime}, e\right)\right) .
$$


Therefore

$$
\lim _{t \rightarrow 0} \frac{d}{d t} \mathcal{E}\left(\varphi_{t}\right)=\sum_{e \in I} \lim _{t \rightarrow 0} \frac{d}{d t} v\left(\varphi_{t}(f, e), \varphi_{t}\left(f^{\prime}, e\right)\right)+\sum_{e \in J} \lim _{t \rightarrow 0} \frac{d}{d t} v\left(\varphi_{t}(f, e), \varphi_{t}\left(f^{\prime}, e\right)\right) .
$$

By Lemma 4.6, every term with index in $I$ is finite while every term with index in $J$ is $\infty$. This is a contradiction. Since we assume $\varphi_{0}$ is the maximum point, $\lim _{t \rightarrow 0} \frac{d}{d t} \mathcal{E}\left(\varphi_{t}\right)$ should be non-positive.

Now we may assume under the angle structure $\varphi_{0}$, there is no Euclidean or degenerated Euclidean triangles. Second we claim that under the angle structure $\varphi_{0}$, there is no zero angle. Otherwise some face $f$ has a triangle $\triangle_{e}$ with angles $(\varphi(f, e)=0, x, \pi-D(e))$. Since the sum $\sum_{e<f} \varphi(f, e)=\frac{1}{2} \Phi(f)>0$, face $f$ has another triangle $\triangle_{e^{\prime}}$ with angles $\left(\varphi\left(f, e^{\prime}\right)=y_{1}>0, y_{2}, \pi-D\left(e^{\prime}\right)\right)$. Now we keep all the angles in the triangles other than $\triangle_{e}$ and $\triangle_{e^{\prime}}$ while modify the angles of $\triangle_{e}$ to $(t, x, \pi-D(e))$ and modify the angles of $\triangle_{e^{\prime}}$ to $\left(y_{1}-t, y_{2}, \pi-D\left(e^{\prime}\right)\right)$. Since there is no Euclidean or degenerated Euclidean triangles in $\varphi_{0}$, then $0+x+\pi-D(e)<\pi$. We can make $t+x+\pi-D(e)<\pi$ for sufficient small $t>0$. And since $y_{1}>0$, we can make $y_{1}-t>0$ for sufficient small $t>0$. Therefore this angle assignment gives a point $\varphi_{t}$ in the closure of $\mathcal{A}$. Now

$$
\lim _{t \rightarrow 0} \frac{d}{d t} \mathcal{E}\left(\varphi_{t}\right)=\lim _{t \rightarrow 0} \frac{d}{d t} v(t, x)+\lim _{t \rightarrow 0} \frac{d}{d t} v\left(y_{1}-t, y_{2}\right) .
$$

Since

we have

$$
\frac{d}{d t} v\left(\varphi_{1}(t), \varphi_{2}(t)\right)=\varphi_{2}^{\prime}(t) \ln \tanh \frac{r_{1}(t)}{2}+\varphi_{1}^{\prime}(t) \ln \tanh \frac{r_{2}(t)}{2},
$$

$$
\lim _{t \rightarrow 0} \frac{d}{d t} v(t, x)=\lim _{t \rightarrow 0}\left[x^{\prime} \ln \tanh \frac{r_{1}(t)}{2}+t^{\prime} \ln \tanh \frac{r_{2}(t)}{2}\right]=\lim _{t \rightarrow 0} \ln \tanh \frac{r_{2}(t)}{2},
$$

where $r_{1}(t)$ (or $r_{2}(t)$ ) is the edge length opposite to angle $\mathrm{t}$ (or $x$ ) in the hyperbolic triangle with angles $(t, x, \pi-D(e))$. Thus $\lim _{t \rightarrow 0} r_{2}(t)=\infty$. Hence $\lim _{t \rightarrow 0} \frac{d}{d t} v(t, x)=0$.

And

$\lim _{t \rightarrow 0} \frac{d}{d t} v\left(y_{1}-t, y_{2}\right)=\lim _{t \rightarrow 0}\left[y_{2}^{\prime} \ln \tanh \frac{s_{1}(t)}{2}+\left(y_{1}-t\right)^{\prime} \ln \tanh \frac{s_{2}(t)}{2}\right]=-\lim _{t \rightarrow 0} \ln \tanh \frac{s_{2}(t)}{2}$, where $s_{1}(t)$ (or $s_{2}(t)$ ) is the edge length opposite to angle $y_{1}-t$ (or $\left.y_{2}\right)$ in the hyperbolic triangle with angles $\left(y_{1}-t, y_{2}, \pi-D\left(e^{\prime}\right)\right)$. Thus $\lim _{t \rightarrow 0} s_{2}(t)$ are finite. Hence $\lim _{t \rightarrow 0} \frac{d}{d t} v\left(y_{1}-t, y_{2}\right)$ is a positive finite number.

This is a contradiction. Since we assume $\varphi_{0}$ is the maximum point, $\lim _{t \rightarrow 0} \frac{d}{d t} \mathcal{E}\left(\varphi_{t}\right)$ should be non-positive.

By the above argument we have show under the angle structure $\varphi_{0}$, every triangle is a hyperbolic triangle. Hence $\varphi_{0} \in \mathcal{A}$. Therefore $\varphi_{0}$ is a critical point of the energy function $\mathcal{E}$. Since $\sum_{e<f} \varphi(f, e)=\frac{1}{2} \Phi(f)$, variables $\{\varphi(f, e)\}$ are not free. By the method of Lagrange multiplier, the angle structure $\left\{\varphi_{0}\right\}$ is a critical point of the energy function if and only if there exists a constant $c_{f}$ for each face $f$ such that

$$
0=\frac{d}{d \varphi(f, e)}\left[\mathcal{E}(\varphi)-\sum_{f \in F} c_{f}\left(\sum_{e<f} \varphi(f, e)-\frac{1}{2} \Phi(f)\right)\right]=\ln \tanh \frac{r(f, e)}{2}-c_{f} .
$$

This shows that the edge length $r(f, e)$ does not depend on any edges of the face $f$ but depends only on the face $f$ itself. Thus we can take it to be the radius of the 
face $f$. This gives us a radius function $r: F \rightarrow(0, \infty)$, equivalently a hyperbolic circle pattern.

By the argument above, a hyperbolic circle pattern induces an angle structure which is a critical point of the energy function $\mathcal{E}$. Since the function $\mathcal{E}$ is strictly concave down in the convex set $\mathcal{A}$, it has a unique critical point which implies the uniqueness of the hyperbolic circle pattern up to isometry.

\section{ACKNOWLEDGEMENT}

The author would like to thank his advisor, Feng Luo, for guidance and encouragement on this work.

\section{REFERENCES}

1. A. I. Bobenko \& B. A. Springborn, Variational principles for circle patterns and Koebe's theorem. Trans. Amer. Math. Soc. 356 (2004), no. 2, 659-689

2. W. Brägger, Kreispackungen und Triangulierungen. Enseign. Math., 38:201-217, 1992

3. Y. Colin de Verdiére, Un principe variationnel pour les empilements de cercles. Invent. Math., 104:655-669, 1991

4. Ren Guo, Geometric angle structures on triangulated surfaces. to appear in Proc. Amer. Math. Soc., arXiv:math.GT/0601486

5. Bernard Kolman \& Robert E. Beck, Elementary Linear Programming with Applications. Second edition, Academic Press, Inc., San Diego, CA, 1995

6. Gregory Leibon, Characterizing the Delaunay decompositions of compact hyperbolic surface. Geom. Topol. 6(2002), 361-391

7. Feng Luo, A characterization of spherical polyhedron surfaces. J. Diff. Geom. 74(2006), no.3, 407-424

8. Feng Luo, Volume and angle structures on 3-manifolds. to appear in Asia Jour. of Math., arXiv:math.GT/0504049

9. Feng Luo, On Teichmuller space of surfaces with boundary. to appear in Duke Math., arXiv:math.GT/0601364

10. Feng Luo, Rigidity of polyhedral surfaces. arXiv:math.GT/0612714

11. Al Marden \& Burt Rodin, On Thurston's formulaton and proof of Andreeev's theorem. Computational methods and function theory (Valparaíso, 1989), 103-116, Lecture Notes in Math., 1435, Springer, Berlin, 1990

12. Igor Rivin, Euclidean structures of simplicial surfaces and hyperbolic volume. Ann. of Math., 139:553-580, 1994

13. Igor Rivin, Combinational optimization in geometry. Advance in Applied Math. 31(2003), no. $1,242-271$

14. B. A. Springborn, Variational principles for circle patterns. PhD thesis, Technische Universität Berlin, 2003. arXiv:math.GT/0312363

15. B. A. Springborn, A variational principle for weighted Delaunay triangulations and hyperideal polyhedra. arXiv:math.GT/0603097

16. W. P. Thurston, Geometry and topology of 3-manifolds. lecture notes of Math Dept. of Princeton University, 1978, avaliable at www.msri.org/publications/books/gt3m/

Department of Mathematics, Rutgers University, Piscataway, NJ, 08854

E-mail address: renguo@math.rutgers.edu 